\title{
Genomic Instability and Clonal Evolution in Chronic Lymphocytic Leukemia: Clinical Relevance
}

\author{
Adalgisa Condoluci, MD, ${ }^{1,2}$ and Davide Rossi, MD, $\mathrm{PhD}^{1,2}$
}

\begin{abstract}
Genomic instability and clonal heterogeneity can influence cancer progression, response to therapy, and relapse. Chronic lymphocytic leukemia (CLL) harbors a variety of clones and subclones that will evolve differently according to intrinsic (microenvironment) and extrinsic (therapy) pressures. Different patterns of clonal evolution have been described, providing insights into the CLL leukemic cell, dynamics, selection, and treatment refractoriness. With the help of genomic technologies allowing a granular resolution of CLL clones, novel synergic therapeutic strategies can be tested with the aim of reaching a genomic-epigenomic ultrapersonalized, tailored approach. These efforts should consider the presence of targetable alterations, continuous cancer reshaping conferring disease refractoriness, and intratumoral clonal equilibrium to possibly avoid clonal selection.
\end{abstract}

J Natl Compr Canc Netw 2021;19(2):227-233 doi: $10.6004 /$ jnccn.2020.7623

${ }^{1}$ Division of Hematology, Oncology Institute of Southern Switzerland, and ${ }^{2}$ Laboratory of Experimental Hematology, Institute of Oncology Research, Bellinzona, Switzerland.
The concept that cancer results from the accumulation of genetic lesions is widely recognized. At a single-cell level, a large amount of alterations benefiting tumor clones over intrinsic (microenvironment, immune system control) and extrinsic (treatment) selective pressures is promoted during cancer evolution. This underlying genetic heterogeneity confers to cancer the capability of readily reshaping the tumor in an evolutionary fashion by either selecting or suppressing subclones for optimization of cancer progression under adverse conditions. ${ }^{1-3} \mathrm{~A}$ permissive landscape with higher tolerance to genomic damage due to genetic instability, mainly linked to the disruption of DNA repair pathways or checkpoints (ie, TP53 or ATM mutations), plays a key role in this setting. ${ }^{4,5}$

\section{Unselected Chronic Lymphocytic Leukemia and Clonal Evolution}

The typical genome of unselected chronic lymphocytic leukemia (CLL) harbors approximately 2,000 molecular lesions, with few recurring at a frequency $>5 \% .{ }^{6,7}$ Conventional karyotyping in the 1990s and fluorescence in situ hybridization (FISH) molecular karyotyping in the 2000s first documented the clonal heterogeneity of CLL by disclosing early, trunk, and fully clonal chromosomal abnormalities, namely deletion 13q14 and trisomy 12, and late, subclonal abnormalities often selected by chemotherapy, namely deletion 11q22-23 and deletion $17 \mathrm{p} 13.8,9$

Deletion 13q14 is the most frequent genetic lesion of CLL, occurring in $50 \%$ to $60 \%$ of cases (Table 1). Deletion of miR15A and miR16A, which favors the constitutive survival and cycling of tumor B cells, is contained within the minimal deleted region on 13q14. These miRNAs physiologically inhibit the expression of key regulators of apoptosis and the cell cycle, ${ }^{10}$ with $B C L 2$ being one of the upregulated genes in CLL as a consequence of $13 \mathrm{q} 14$ deletion. ${ }^{11}$

$A T M$ is a member of the phosphatidylinositol 3-kinase gene family, which regulates DNA damageinduced cell cycle arrest at G1/S and G2/M by activating DNA repair pathways and by inducing apoptosis whenever the DNA damage cannot be repaired. ATM maps 


\begin{tabular}{|lcc|}
\hline $\begin{array}{c}\text { Table 1. Clinical Relevance of Recurrent Genetic } \\
\text { Alterations in CLL }\end{array}$ & $\begin{array}{c}\text { Prevalence at } \\
\text { Diagnosis }\end{array}$ & $\begin{array}{c}\text { Prevalence at } \\
\text { Progression/Relapse }\end{array}$ \\
\hline Genetic Lesion & $50 \%-60 \%$ & $\sim 50 \%-60 \%$ \\
\hline $13 q 14$ deletion & $15 \%-20 \%$ & $\sim 20 \%$ \\
\hline Trisomy 12 & $8 \%-9 \%$ & $30 \%$ \\
\hline $11 q 22-q 23$ deletion & $4 \%-5 \%$ & $30 \%$ \\
\hline $17 p 13$ deletion & $40 \%$ & $70 \%-80 \%$ \\
\hline IGHV lack of mutation & $5 \%-10 \%$ & $30 \%-40 \%$ \\
\hline TP53 mutation & $4 \%-10 \%$ & $30 \%$ \\
\hline NOTCH1 mutation & $6 \%-9 \%$ & $20 \%$ \\
\hline SF3B1 mutation & $10 \%-15 \%$ & $15 \%-20 \%$ \\
\hline ATM mutation & & \\
\hline
\end{tabular}

Abbreviations: CLL, chronic lymphocytic leukemia; IGHV, immunoglobulin heavy variable.

on chromosome 11q22-q23 and may be inactivated by both deletion and somatic mutations in CLL. ${ }^{12-14}$ Only $10 \%$ to $20 \%$ of $A T M$-mutated CLL cases harbor a concomitant 11q22-q23 deletion, and only $20 \%$ to $30 \%$ of CLL cases with 11q22-q23 deletion also have concurrent ATM mutations. ${ }^{15}$

Trisomy 12 is reported in approximately $16 \%$ of cases of CLL and has a higher prevalence (28\%) in atypical phenotypes of CLL and small lymphocytic lymphoma. ${ }^{16}$ Trisomy 12 usually correlates to an increased prevalence of NOTCH1 mutations (25\%-30\%) and unmutated immunoglobulin heavy variable (IGHV) genes. ${ }^{17}$

Deletions of 17p13/ TP53 mutations occur in $10 \%$ of newly diagnosed CLL and progressive CLL requiring first treatment, and in $30 \%$ to $40 \%$ of cases of relapsed CLL (Table 1). The TP53 gene may be inactivated by deletion and/or somatic mutations. ${ }^{18,19}$ Because of the genetic instability associated with defective DNA damage checkpoints, complex cytogenetic abnormalities (eg, unbalanced translocations) are frequently concurrent with TP53 abnormalities..$^{20-23}$

\section{Clustering and Timing of Clonal Evolution}

The concept of clonal evolution has been extended within genomic studies based on next-generation sequencing. Recurrent mutations affecting genes involved in the development and progression of CLL can be clustered in a small set of pathways, including microenvironment-dependent signaling ( $\mathrm{NOTCH1}$, FBXW7), inflammatory receptors (MYD88), mitogenactivated protein kinase/extracellular signal-regulated kinase (BRAF, KRAS, NRAS, MAP2K1), and nuclear factor kappa B (NF-кB) pathways (BIRC3, TRAF3, NFKBIE), and also as intracellular programs, such as DNA damage and cell cycle control (ATM, TP53, SAMHD1, POT1), chromatin modification (HIST1H1E, CHD2, ZMYM3), transcription (EGR2, IRF4, BCOR, MED12), and RNA processing (XPO1, SF3B1, RPS15). ${ }^{6,7}$

Deletion 13q14 and trisomy 12 are similarly represented in all phases of the disease, and are therefore characterized as first-step genetic abnormalities in CLL (Table 1). ${ }^{24-27}$ The other genetic events usually represent second-hit alterations that are progressively selected or acquired during clonal evolution and that occur in a more advanced phase of the disease. Based on conventional and FISH cytogenetic analysis, clonal evolution mainly consists in the development of $17 \mathrm{p} 13$ or 11q22-q23 deletions (Table 1). The development of new TP53 mutations also contributes to clonal evolution, especially in patients with chemotherapy-refractory CLL and those with Richter syndrome. ${ }^{28,29}$ SF3B1, $A T M$, and BIRC3 mutations, which correlate to a more aggressive clinical phenotype, may emerge during the course of CLL, thus expanding the spectrum of genetic events associated with clonal evolution (Table 1)..$^{27-29}$ The greater the intrapatient clonal heterogeneity of CLL, the worse the patient's outcome. The unfavorable outcome of patients carrying subclonal driver alterations (mutations and/or copy number alterations [CNAs]) has been consistently documented by both considering single genetic abnormalities and the whole spectrum of coding mutations. $^{23,30,31}$

Treatment of patients with CLL is currently guided according to different characteristics of the patient (fitness, comorbidities, symptoms) and the disease (treatment timeline, clinical stage, genetics). Some molecular findings are described as predictive biomarkers, providing information on the likely benefit of a specific treatment. ${ }^{32}$ One of these biomarkers is the IGHV mutational status. Intensive chemoimmunotherapy (CIT; eg, fludarabine/cyclophosphamide/rituximab) is still an option for young (aged $<65$ years), fit patients with mutated IGHV genes, because it is reported that $50 \%$ to $60 \%$ of these patients maintain disease remission in the long term-including persistent negativity of minimal residual disease in some instances, showing no relapses beyond 10 years - and have an overall survival similar to that of healthy subjects. ${ }^{33-36}$ Conversely, in the long term, almost all patients with IGHV-unmutated CLL are going to experience disease progression after CIT. ${ }^{33-36}$ Patients with CLL benefit from novel agents independent of IGHV mutation status. ${ }^{37-39}$ TP53 disruption (deletions of the short arm of chromosome 17 and/or mutations of the TP53 gene) predicts resistance to CIT. ${ }^{40-43}$ Because their antileukemic efficacy is not exerted through genotoxic mechanisms, novel agents (ibrutinib/acalabrutinib, idelalisib/duvelisib, and venetoclax) are active independently of TP53 dysfunction..$^{44}$ Accordingly, guidelines for management of CLL recommend testing for $17 \mathrm{p} 13$ deletion and TP53 mutations in patients in need of 
treatment, ${ }^{45}$ and targeted therapy is indicated for patients with a disease harboring this predictive biomarker.

\section{Patterns of Clonal Evolution}

Sequential sampling of peripheral blood collected at different time points (ie, from pretreatment to relapse after treatment) from patients with CLL helped to describe clonal dynamic patterns over time (Table 2). Some statistical tools have also been developed to identify the subclonal driver events (eg, PhylogicNDT; Broad Institute of MIT and Harvard), and clonal interaction can be traced by ClonTracer (Novartis Institute for Biomedical Research), GESTALT (University of Washington and Harvard), and COLBERT (The University of Texas at Austin). Different patterns of clonal evolution have been described, along with the associated clinical relevance. ${ }^{46-50}$ Despite efforts to describe the evolutionary events in CLL, the direct influence of these events on an adapted treatment strategy (and vice versa) is not currently fully embraced.

\section{Clonal Equilibrium and Competition}

Clonal equilibrium is characterized by the coexistence of a mixed and balanced leukemic population over time. Clonal equilibrium typically occurs in the absence of a strong selection pressure, such as treatment, and during progression from the monoclonal B-cell lymphocytosis to overt CLL (Figure 1). ${ }^{23,30}$ Clonal competition is characterized by $\geq 1$ clones that are more prone to proliferate according to their genetic alterations (Figure 2). In this context, it has recently been shown that the CLL lineage tree shape is characterized by earlier branching and longer branch lengths than normal $B$ cells, reflecting rapid drift after the initial malignant transformation and a greater proliferative history. ${ }^{51}$

\section{No Evolution}

In most patients, CNAs and/or mutations in putative driver genes were identified before therapy. ${ }^{52}$ Patients lacking clonal evolution with persistent subclones over time and treatment, and thus depicting a stable genetics, usually show a more indolent disease..$^{23}$ In one study, $90 \%$ of untreated patients with CLL did not show clonal evolution, whereas up to $52 \%$ of treated patients developed clonal changes. This observation suggests that treatment facilitates the expansion of clones, which are destined to become dominant by removing their competitors, ${ }^{29}$ and supports the watch-and-wait approach in the absence of clear treatment indications to avoid accelerating clonal evolution and the selection of more aggressive subclones.

\section{Linear Evolution}

Linear evolution is defined as the persistence of a founder clone, with further acquisition of new mutations. Linear evolution is common in Richter syndrome

\section{Table 2. Genomic Studies of Clonal Evolution in CLL}

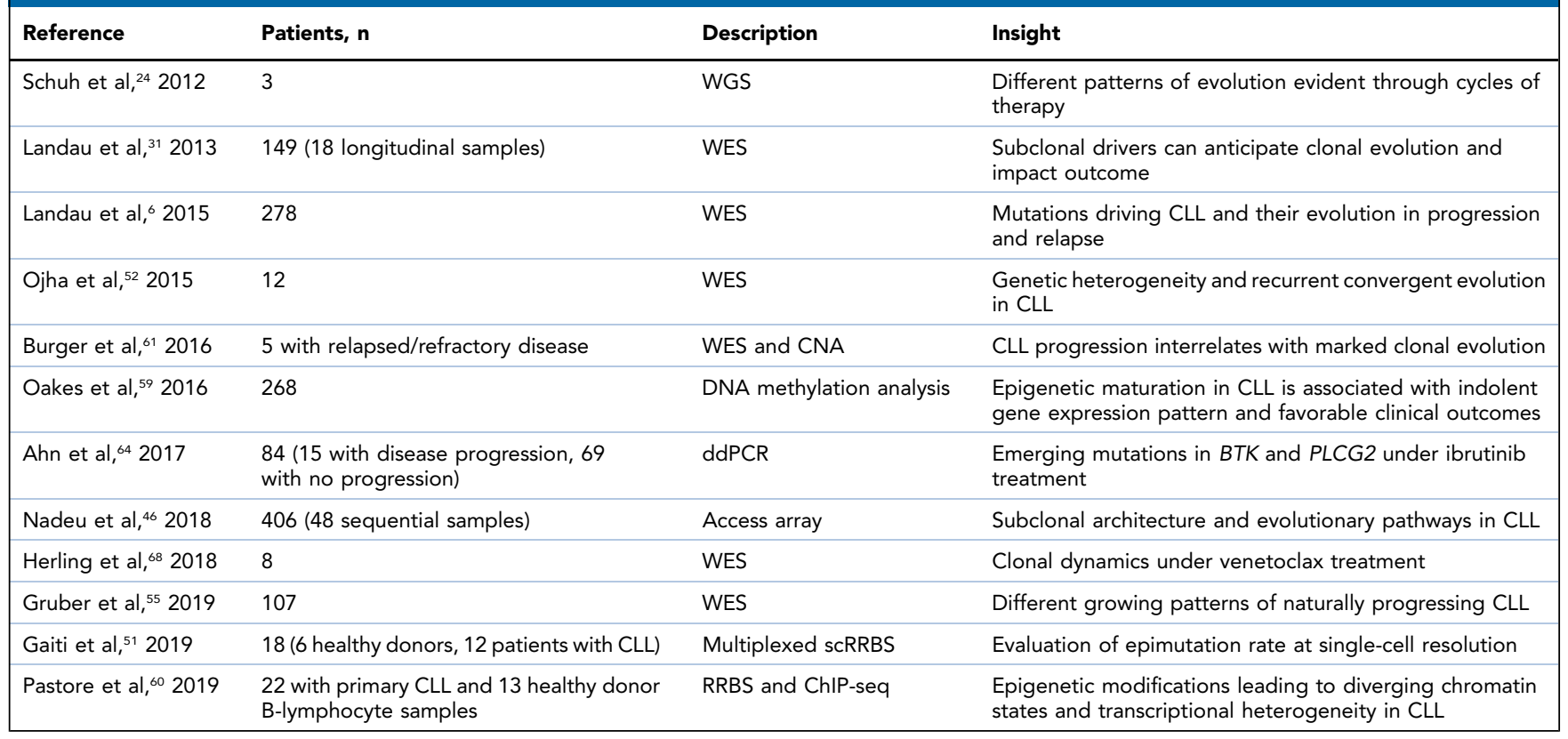

Abbreviations: ChIP-seq, chromatin immunoprecipitation sequencing; CLL, chronic lymphocytic leukemia; CNA, copy number analysis; ddPCR, droplet digital PCR; RRBS, reduced-representation bisulfite sequencing; scRRBS, single-cell reduced-representation bisulfite sequencing; WES, whole-exome sequencing; WGS, whole-genome sequencing. 


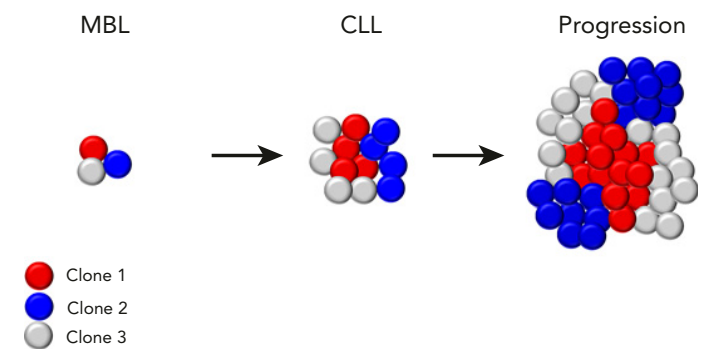

Figure 1. Clonal equilibrium model: a model of CLL progression in the absence of selection representing 3 subclones proportionally growing over time until eventual progression.

Abbreviations: CLL, chronic lymphocytic leukemia; MBL, monoclonal B-cell lymphocytosis.

transformation (90\% of cases), but not in CLL progression after CIT (33\% of cases) or during ibrutinib therapy $(7 \%){ }^{29,52,53}$

\section{Branching Evolution}

Branching evolution is defined as the parallel evolution of competitive clones. Multibranching evolution with $\geq 2$ subclones fluctuating over time is common during CIT ( $67 \%$ of patients) or ibrutinib therapy ( $25 \%$ of patients)..$^{52,53}$

\section{Convergent Mutational Evolution}

Convergent mutational evolution, defined as the acquisition of $\geq 1$ mutation in the same gene, has been reported as a common phenomenon in CLL drivers. Convergent mutational evolution was described in up to $26 \%$ of patients and affected nearly $70 \%$ of the CLL driver genes. ${ }^{46,52,54}$

\section{Logistic Growth and Exponential Growth}

Gruber et $\mathrm{al}^{55}$ described the clonal evolution of 107 patients with untreated CLL after collecting blood samples from diagnosis to first treatment, documenting that different CLL cases can exhibit diverse growth patterns. The logistic-like pattern is sigmoidal and achieves a potentially temporary stable state with $<1,000 \times 10^{9}$ cells. Patients showing this growth pattern have a disease that eventually will never require therapy after diagnosis, harboring genetically favorable features such as deletion 13q14 and mutated IGHV status. Conversely, the exponential pattern shows a higher genetic complexity and a faster disease progression, with $>1,000 \times 10^{9}$ cells. $^{55}$ This observation mandates caution regarding treatment initiation based on lymphocyte increase or doubling time alone in the context of modest absolute peripheral blood lymphocytosis, because some of those patients are destined to stabilize tumor burden and may not ultimately require therapy.

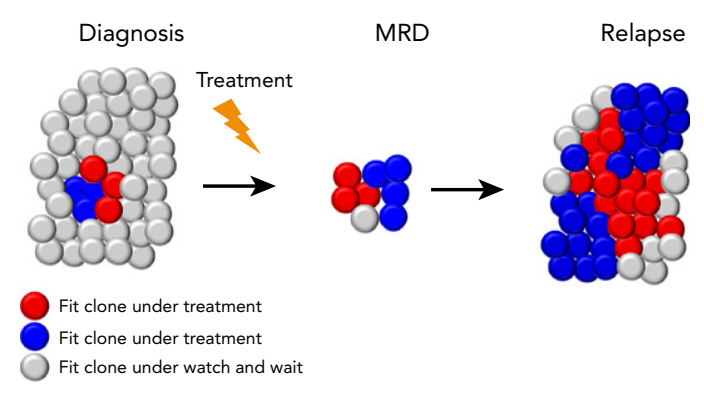

Figure 2. Clonal competition model: clonal evolution under treatment selection pressure. At diagnosis, the fittest clone for watch and wait predominates over other subclones. After treatment, resistant clones are selected and the MRD clones expand upon relapse. Abbreviation: MRD, minimal residual disease.

\section{Role of Epigenetics in Clonal Reshaping}

Epigenetics, including DNA methylation, chromatin remodeling, and posttranslational histone modification, can affect genomic stability by determining the biologic state of the cells. These mechanisms allow cancer cells to quickly adapt to environmental stressors, including therapy and immune editing. Genome-wide arrays and sequencing allowed the definition of the epigenetic landscape of CLL as generally stable over time, sharing common features with normal B-cell differentiation across resting and proliferative compartments. ${ }^{56-58}$ Nevertheless, a more pronounced epigenetic heterogeneity has been described in CLL cells with respect to normal CD19-positive lymphocytes. This heterogeneity could support genetic instability, providing a potential propensity to develop alternative evolutionary trajectories under extrinsic or intrinsic clonal pressure. ${ }^{51,58,59}$ Epigenetic heterogeneity has been reported as correlating with worse outcome and more aggressive disease. ${ }^{51,60}$

\section{Clonal Evolution Upon Targeted Treatment}

Gene mutations are well-known mechanisms of resistance to targeted therapy in CLL. Burger et $\mathrm{al}^{61}$ suggested that time to clinically detectable relapse after ibrutinib therapy is determined not only by the presence of resistanceconferring mutations within subclonal populations but also by the size and growth rate of the drug-resistant clone at the time of treatment initiation (Figure 3).

Acquisition of $B T K$ mutations at the binding site of ibrutinib (ie, Cys481Ser) or in the SH2 domain of BTK (T316A), or of gain-of-function mutations in phospholipase C $\gamma 2$ (PLCG2) has been described in up to $85 \%$ of patients developing resistance to ibrutinib. ${ }^{61,62}$ Detection of such mutations can occur up to 15 months before clinical relapse ${ }^{61}$ conferring a predictive role to these biomarkers, which warrants further prospective data collection. Other lesions associated with ibrutinib resistance include $8 p$ deletion, which encompasses the TRAIL receptor, conferring resistance to TRAIL-induced apoptosis; gain-of-function mutation of CARD11, eventually activating the NF-кB 


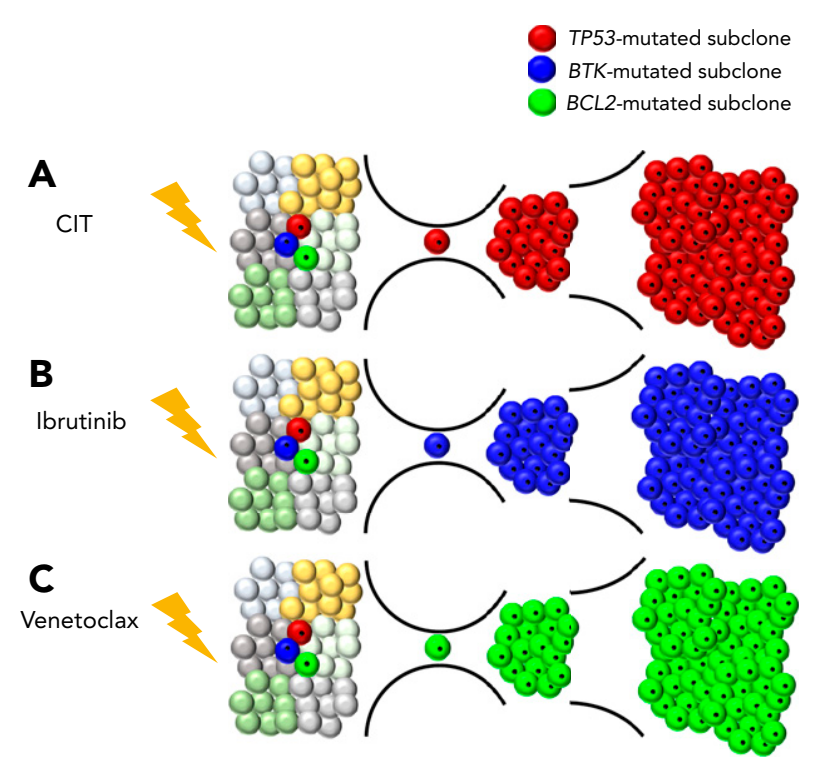

Figure 3. Clonal architecture of resistance clones: selection of resistant clones under different treatment regimens. (A) TP53-mutated subclones leading to resistance/progression under CIT. (B) BTK-mutated subclones leading to resistance/progression under ibrutinib. (C) BCL2mutated subclones leading to resistance/progression under venetoclax. Abbreviation: $\mathrm{CIT}$, chemoimmunotherapy.

pathway downstream from Bruton tyrosine kinase; and mutations of $I T P K B$, a central feedback inhibitor of the B-cell receptor signaling pathway. ${ }^{39,63-66}$

Mutations are a mechanism of resistance to venetoclax therapy, at least in a proportion of cases. A single-nucleotide variant in BCL2 (Gly101Val), the target of venetoclax, affects the binding of the drug to the protein, occurs in up to $50 \%$ of cases developing acquired resistance, and can be detected up to 25 months earlier than standard disease progression criteria are met. ${ }^{67}$ Herling et $\mathrm{al}^{68}$ reported that a progressively increasing number of acquired CNAs or aneuploidy occurs upon venetoclax treatment, showing signs of accumulating genomic instability in CLL surviving cells. Mutations in BTG1 and homozygous deletions of CDKN2A/ $B$ were reported as recurrent genetic alterations in patients with venetoclax-refractory CLL. ${ }^{1}$

\section{Conclusions}

A new concept of ultrapersonalized medicine based on knowledge of the genomic-epigenomic structure of CLL could be developed. The goals of such a strategy could be targeting the specific genetic or epigenetic lesions that
A

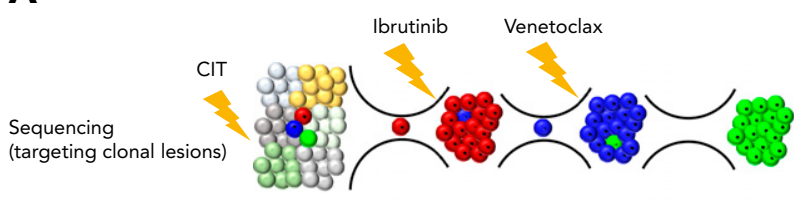

B

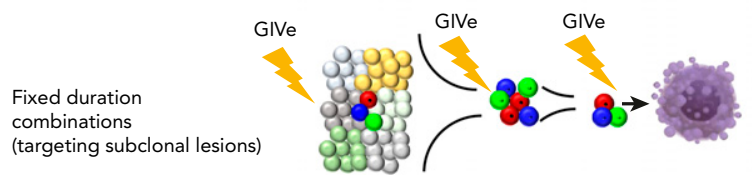

Figure 4. Proposal of a genomic-oriented approach targeting clonal versus subclonal lesions. (A) A sequential approach starting with CIT would eventually select TP53-disrupted clones, which could be targeted with the introduction of ibrutinib, eventually selecting BTKmutated subclones. Ibrutinib-resistant subclones could be overcome with venetoclax. (B) A fixed-duration combination of different agents (eg, GIVe) would target a wide range of subpopulations, allowing the possibility to overcome potential resistance mechanisms.

Abbreviations: CIT, chemoimmunotherapy; GIVe, obinutuzumab/ibrutinib/venetoclax.

can confer an aggressive or resistant phenotype to a clone, even sparing the more stable clones, and reducing the heterogeneity of the disease and the consequent loss of effectiveness of a targeted treatment, applying a debulking approach that may act as an evolutionary restriction point, further resetting interclonal dynamics. A genomic-oriented approach targeting the disease at a clonal-subclonal level is proposed in Figure 4 and could follow a sequential approach (ie, starting with CIT, which would eventually select TP53-disrupted clones, then introducing ibrutinib to overcome TP53 mutations but eventually selecting BTK mutations, and finally switching to venetoclax upon development of ibrutinib-resistant mutations). Nevertheless, a combination of different agents would target a wide range of subpopulations, allowing the ability to overcome potential resistance mechanisms.

Submitted January 2, 2020; accepted for publication July 15, 2020. Published online December 31, 2020.

Disclosures: The authors have disclosed that they have no financial interests, arrangements, affiliations, or commercial interests with the manufacturers of any products discussed in this article or their competitors.

Correspondence: Davide Rossi, MD, PhD, Division of Hematology, Oncology Institute of Southern Switzerland, and Laboratory of Experimental Hematology, Institute of Oncology Research, Via A. Gallino 13, 6500 Bellizona, $\mathrm{CH}$ Bellinzona, Switzerland. Email: davide.rossi@eoc.ch

\section{References}

1. Nowell PC. The clonal evolution of tumor cell populations. Science 1976; 194:23-28.

2. Fidler IJ. Tumor heterogeneity and the biology of cancer invasion and metastasis. Cancer Res 1978;38:2651-2660.

3. Merlo LM, Pepper JW, Reid BJ, et al. Cancer as an evolutionary and ecological process. Nat Rev Cancer 2006;6:924-935.
4. Bouwman P, Jonkers J. The effects of deregulated DNA damage signalling on cancer chemotherapy response and resistance. Nat Rev Cancer 2012;12:587-598.

5. Bassaganyas L, Beà S, Escaramís G, et al. Sporadic and reversible chromothripsis in chronic lymphocytic leukemia revealed by longitudinal genomic analysis. Leukemia 2013;27:2376-2379. 
6. Landau DA, Tausch E, Taylor-Weiner AN, et al. Mutations driving CLL and their evolution in progression and relapse. Nature 2015;526:525-530

7. Puente $X S$, Beà $S$, Valdés-Mas $R$, et al. Non-coding recurrent mutations in chronic lymphocytic leukaemia. Nature 2015;526:519-524.

8. Juliusson G, Oscier DG, Fitchett M, et al. Prognostic subgroups in B-cell chronic lymphocytic leukemia defined by specific chromosomal abnormalities. N Engl J Med 1990;323:720-724.

9. Döhner H, Stilgenbauer S, Benner A, et al. Genomic aberrations and survival in chronic lymphocytic leukemia. N Engl J Med 2000;343: 1910-1916.

10. Calin GA, Dumitru CD, Shimizu M, et al. Frequent deletions and down-regulation of micro-RNA genes miR15 and miR16 at 13q14 in chronic lymphocytic leukemia. Proc Natl Acad Sci USA 2002;99: 15524-15529.

11. Roberts AW, Davids MS, Pagel JM, et al. Targeting BCL2 with venetoclax in relapsed chronic lymphocytic leukemia. N Engl J Med 2016;374: 311-322.

12. Sperka T, Wang J, Rudolph KL. DNA damage checkpoints in stem cells, ageing and cancer. Nat Rev Mol Cell Biol 2012;13:579-590.

13. Schaffner $C$, Stilgenbauer $S$, Rappold GA, et al. Somatic ATM mutations indicate a pathogenic role of ATM in B-cell chronic lymphocytic leukemia. Blood 1999;94:748-753.

14. Stankovic $T$, Weber $P$, Stewart $G$, et al. Inactivation of ataxia telangiectasia mutated gene in B-cell chronic lymphocytic leukaemia. Lancet 1999;353: 26-29.

15. Ouillette $P$, Li J, Shaknovich R, et al. Incidence and clinical implications of ATM aberrations in chronic lymphocytic leukemia. Genes Chromosomes Cancer 2012;51:1125-1132.

16. Que TH, Marco JG, Ellis J, et al. Trisomy 12 in chronic lymphocytic leukemia detected by fluorescence in situ hybridization: analysis by stage, immunophenotype, and morphology. Blood 1993;82:571-575.

17. Balatti V, Bottoni A, Palamarchuk $A$, et al. NOTCH1 mutations in CLL associated with trisomy 12. Blood 2012;119:329-331.

18. Rossi $D$, Cerri $M$, Deambrogi $C$, et al. The prognostic value of TP53 mutations in chronic lymphocytic leukemia is independent of Del17p13: implications for overall survival and chemorefractoriness. Clin Cancer Res 2009;15:995-1004.

19. Malcikova J, Smardova J, Rocnova L, et al. Monoallelic and biallelic inactivation of TP53 gene in chronic lymphocytic leukemia: selection, impact on survival, and response to DNA damage. Blood 2009;114: 5307-5314.

20. Rossi $D$, Khiabanian $H$, Spina $V$, et al. Clinical impact of small TP53 mutated subclones in chronic lymphocytic leukemia. Blood 2014;123: 2139-2147

21. Baliakas $P$, Hadzidimitriou $A$, Sutton $L A$, et al. Recurrent mutations refine prognosis in chronic lymphocytic leukemia. Leukemia 2015;29:329-336.

22. International CLL-IPI Working Group. An international prognostic index for patients with chronic lymphocytic leukaemia (CLL-IPI): a meta-analysis of individual patient data. Lancet Oncol 2016;17:779-790.

23. Nadeu F, Delgado J, Royo $C$, et al. Clinical impact of clonal and subclonal TP53, SF3B1, BIRC3, NOTCH1, and ATM mutations in chronic lymphocytic leukemia. Blood 2016;127:2122-2130

24. Schuh A, Becq J, Humphray S, et al. Monitoring chronic lymphocytic leukemia progression by whole genome sequencing reveals heterogeneous clonal evolution patterns. Blood 2012;120:4191-4196.

25. Rawstron AC, Bennett FL, O'Connor SJ, et al. Monoclonal B-cell lymphocytosis and chronic lymphocytic leukemia. N Engl J Med 2008;359: 575-583.

26. Rossi D, Sozzi E, Puma A, et al. The prognosis of clinical monoclonal $B$ cell lymphocytosis differs from prognosis of Rai 0 chronic lymphocytic leukaemia and is recapitulated by biological risk factors. Br J Haematol 2009; 146:64-75.

27. Kern W, Bacher U, Haferlach C, et al. Monoclonal B-cell lymphocytosis is closely related to chronic lymphocytic leukaemia and may be better classified as early-stage CLL. Br J Haematol 2012;157:86-96.

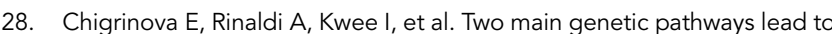
the transformation of chronic lymphocytic leukemia to Richter syndrome. Blood 2013;122:2673-2682.

29. Fabbri G, Khiabanian H, Holmes AB, et al. Genetic lesions associated with chronic lymphocytic leukemia transformation to Richter syndrome. J Exp Med 2013;210:2273-2288.

30. Ouillette P, Saiya-Cork K, Seymour E, et al. Clonal evolution, genomic drivers, and effects of therapy in chronic lymphocytic leukemia. Clin Cancer Res 2013;19:2893-2904.
31. Landau DA, Carter SL, Stojanov P, et al. Evolution and impact of subclonal mutations in chronic lymphocytic leukemia. Cell 2013;152:714-726.

32. Ballman KV. Biomarker: predictive or prognostic? J Clin Oncol 2015;33: 3968-3971.

33. Rossi D, Terzi-di-Bergamo L, De Paoli L, et al. Molecular prediction of durable remission after first-line fludarabine-cyclophosphamiderituximab in chronic lymphocytic leukemia. Blood 2015;126: 1921-1924.

34. Fischer K, Bahlo J, Fink AM, et al. Long-term remissions after FCR chemoimmunotherapy in previously untreated patients with CLL: updated results of the CLL8 trial. Blood 2016;127:208-215.

35. Thompson PA, Tam CS, O'Brien SM, et al. Fludarabine, cyclophosphamide, and rituximab treatment achieves long-term disease-free survival in IGHV-mutated chronic lymphocytic leukemia. Blood 2016; 127:303-309

36. Eichhorst B, Fink AM, Bahlo J, et al. First-line chemoimmunotherapy with bendamustine and rituximab versus fludarabine, cyclophosphamide, and rituximab in patients with advanced chronic lymphocytic leukaemia (CLL10): an international, open-label, randomised, phase 3, non-inferiority trial. Lancet Oncol 2016;17:928-942.

37. Coutre SE, Leonard JP, Barrientos JC, et al. Clinical activity of idelalisib (GS-1101), a selective inhibitor of PI3Kס, in phase 1 and 2 trials in chronic lymphocytic leukemia (CLL): effect of del(17p)/TP53 mutation, del(11q), IGHV mutation, and NOTCH1 mutation [abstract]. Blood 2013:122:1632. Abstract 642.

38. O'Brien S, Furman RR, Coutre $\mathrm{S}$, et al. Single-agent ibrutinib in treatmentnaive and relapsed/refractory chronic lymphocytic leukemia: a 5-year experience. Blood 2018;131:1910-1919.

39. Burger JA, Tedeschi A, Barr PM, et al. Ibrutinib as initial therapy for patients with chronic lymphocytic leukemia. N Engl J Med 2015;373: 2425-2437.

40. Hallek M, Fischer K, Fingerle-Rowson G, et al. Addition of rituximab to fludarabine and cyclophosphamide in patients with chronic lymphocytic leukaemia: a randomised, open-label, phase 3 trial. Lancet 2010;376: 1164-1174.

41. Fischer $\mathrm{K}$, Cramer $\mathrm{P}$, Busch $\mathrm{R}$, et al. Bendamustine in combination with rituximab for previously untreated patients with chronic lymphocytic leukemia: a multicenter phase II trial of the German Chronic Lymphocytic Leukemia study group. J Clin Oncol 2012;30:3209-3216.

42. Goede V, Fischer K, Busch R, et al. Obinutuzumab plus chlorambucil in patients with CLL and coexisting conditions. N Engl J Med 2014;370: 1101-1110.

43. Hillmen P, Robak T, Janssens A, et al. Chlorambucil plus ofatumumab versus chlorambucil alone in previously untreated patients with chronic lymphocytic leukaemia (COMPLEMENT 1): a randomised, multicentre, open-label phase 3 trial. Lancet 2015;385:1873-1883.

44. Anderson MA, Deng J, Seymour JF, et al. The BCL2 selective inhibitor venetoclax induces rapid onset apoptosis of CLL cells in patients via a TP53-independent mechanism. Blood 2016;127:3215-3224.

45. Hallek M, Cheson BD, Catovsky D, et al. iwCLL guidelines for diagnosis, indications for treatment, response assessment, and supportive management of CLL. Blood 2018;131:2745-2760.

46. Nadeu F, Clot G, Delgado J, et al. Clinical impact of the subclonal architecture and mutational complexity in chronic lymphocytic leukemia. Leukemia 2018;32:645-653.

47. Al'Khafaji AM, Deatherage D, Brock A. Control of lineage-specific gene expression by functionalized gRNA barcodes. ACS Synth Biol 2018;7: 2468-2474.

48. Bhang HE, Ruddy DA, Krishnamurthy Radhakrishna V, et al. Studying clonal dynamics in response to cancer therapy using high-complexity barcoding. Nat Med 2015;21:440-448.

49. Hata AN, Niederst MJ, Archibald HL, et al. Tumor cells can follow distinct evolutionary paths to become resistant to epidermal growth factor receptor inhibition. Nat Med 2016;22:262-269.

50. McKenna A, Findlay GM, Gagnon JA, et al. Whole-organism lineage tracing by combinatorial and cumulative genome editing. Science 2016; 353:aaf7907.

51. Gaiti F, Chaligne R, Gu H, et al. Epigenetic evolution and lineage histories of chronic lymphocytic leukaemia. Nature 2019;569:576-580.

52. Ojha J, Ayres J, Secreto C, et al. Deep sequencing identifies genetic heterogeneity and recurrent convergent evolution in chronic lymphocytic leukemia. Blood 2015;125:492-498.

53. Landau DA, Sun C, Rosebrock D, et al. The evolutionary landscape of chronic lymphocytic leukemia treated with ibrutinib targeted therapy. Nat Commun 2017:8:2185. 
54. Jethwa A, Hüllein J, Stolz T, et al. Targeted resequencing for analysis of clonal composition of recurrent gene mutations in chronic lymphocytic leukaemia. Br J Haematol 2013;163:496-500.

55. Gruber M, Bozic I, Leshchiner I, et al. Growth dynamics in naturally progressing chronic lymphocytic leukaemia. Nature 2019;570: 474-479.

56. Cahill N, Bergh AC, Kanduri M, et al. 450K-array analysis of chronic lymphocytic leukemia cells reveals global DNA methylation to be relatively stable over time and similar in resting and proliferative compartments. Leukemia 2013;27:150-158

57. Kulis $M$, Merkel $A$, Heath $S$, et al. Whole-genome fingerprint of the DNA methylome during human B cell differentiation. Nat Genet 2015;47: 746-756.

58. Beekman R, Chapaprieta V, Russiñol N, et al. The reference epigenome and regulatory chromatin landscape of chronic lymphocytic leukemia. Nat Med 2018;24:868-880.

59. Oakes CC, Seifert M, Assenov Y, et al. DNA methylation dynamics during $B$ cell maturation underlie a continuum of disease phenotypes in chronic lymphocytic leukemia. Nat Genet 2016;48:253-264.

60. Pastore A, Gaiti F, Lu SX, et al. Corrupted coordination of epigenetic modifications leads to diverging chromatin states and transcriptional heterogeneity in CLL. Nat Commun 2019;10:1874.
61. Burger JA, Landau DA, Taylor-Weiner A, et al. Clonal evolution in patients with chronic lymphocytic leukaemia developing resistance to BTK inhibition. Nat Commun 2016;7:11589.

62. Woyach JA, Ruppert AS, Guinn D, et al. BTK ${ }^{\text {C481S }}$-mediated resistance to ibrutinib in chronic lymphocytic leukemia. J Clin Oncol 2017;35: 1437-1443.

63. Lazarian G, Guièze R, Wu CJ. Clinical implications of novel genomic discoveries in chronic lymphocytic leukemia. J Clin Oncol 2017;35:984-993.

64. Ahn IE, Underbayev C, Albitar A, et al. Clonal evolution leading to ibrutinib resistance in chronic lymphocytic leukemia. Blood 2017;129. 1469-1479.

65. Woyach JA, Johnson AJ. Targeted therapies in CLL: mechanisms of resistance and strategies for management. Blood 2015;126:471-477.

66. Kadri S, Lee J, Fitzpatrick $C$, et al. Clonal evolution underlying leukemia progression and Richter transformation in patients with ibrutinib-relapsed CLL. Blood Adv 2017;1:715-727.

67. Blombery $\mathrm{P}$, Anderson MA, Gong JN, et al. Acquisition of the recurrent Gly101Val mutation in BCL2 confers resistance to venetoclax in patients with progressive chronic lymphocytic leukemia. Cancer Discov 2019;9:342-353.

68. Herling CD, Abedpour N, Weiss J, et al. Clonal dynamics towards the development of venetoclax resistance in chronic lymphocytic leukemia. Nat Commun 2018;9:727 